\title{
Research on the Application of Analogy Method in Electromagnetism Teaching
}

\author{
Chen Bingbing \\ Sichuan Minzu College \\ Kangding, Sichuan
}

\begin{abstract}
Electromagnetism is a very important basic course for physics major. Combining with the needs of school application transformation and teaching experience, the idea of analogy method is introduced into the teaching of electromagnetism. Students' understanding and memory on the knowledge can be promoted by comparing the basic concepts, basic rules and some important theorems of electrostatic field and steady magnetic field. And such method can have double effects on the teaching.
\end{abstract}

Keywords-analogy method; electrostatic field; steady magnetic field; teaching

\section{INTRODUCTION}

Electromagnetism is a significant basic course for physics majors, which is an important part of classical physics. It is also closely related to many fields of modern natural science and engineering technology, and it has become an indispensable basis for studying natural materials. On the one hand, students can comprehensively and systematically understand the basic phenomena, concepts and laws of electromagnetic motion after studying this course, so as to have certain ability to analyze and solve electromagnetic problems and lay a necessary foundation for the study of subsequent courses. On the other hand, students can understand physics thoughts and experimental methods, and establish a scientific world view and methodology through the study of electromagnetics system.

\section{THE ROLE AND SIGNIFICANCE OF ANALOGY IN TEACHING}

Analogy method refers to a kind of common attribute of a certain kind of things, which can infer that similar things also have this attribute. Generally speaking, the more attributes an object has in common, and the more reliable the conclusion is.

\section{A. Function of analogy method in electromagnetism teaching}

When studying electromagnetics, students generally reflect that there are many formulas and abstract concepts. The calculation process involves vector method and calculus, so it is rather difficult to learn. However, most of the course content has a strong similarity and symmetry, such as the electrostatic field in the first part and the steady magnetic field in the second part in the course. Although the vector field of two different natures is introduced, they have high similarity and symmetry on the order arrangement of knowledge points, physical concept, the law of theorem and problem-solving

This paper belongs to the Electromagnetism, a school-level demonstration course of Sichuan Minzu College. Project number: sfkc201707 staged achievements. ideas and methods. Therefore, we can timely and appropriately introduce the analogy idea in the teaching. On the one hand, students can know the structure of section knowledge and establish knowledge structure system so as to avoid the confusion of memory due to many things they have learned and high similarity. It is conductive to deepen their understanding, mastering and migrating of knowledge; on the other hand, the introduction of the analogy thought can expand their thinking ways, cultivate their thinking abilities, and stimulate the enthusiasm of actively learning, so as to improve the efficiency of teaching.

\section{B. The significance of analogy in teaching}

1) It is helpful for teachers to better understand the textbook

It is the basis and premise of a good physics lesson that teachers fully understand the teaching material. In order to teach with a clear thought and make students acquire the knowledge better, teachers should fully master the teaching content and knowledge points, straighten out the whole course section and the relationship between the knowledge points and establish a clear knowledge structure in the former preparation and teaching design. However, teachers can use the idea of analogy to establish a clear knowledge system. In order to establish a clear knowledge system, teachers can use the idea of analogy. When dealing with the content of electrostatic field, they only need to grasp the knowledge points such as Coulomb law, Gauss theorem and Ampere circuital theorem. Then when it comes to the content of steady magnetic field, we can use the analogy method to cope with it so as to achieve the double effect.

2) It is conducive to understand and master abstract concepts

There are many formulas and abstract concepts in the course of electromagnetism, which makes it more difficult for students to learn. When dealing with physics problems, we often abstract some physical concepts into physical models to simplify the problems, in order to facilitate our processing and get approximate conclusions. In electromagnetics, all of these are physical models introduced to deal with corresponding problems, such as point charge, exploratory charge, micro-element charge, infinite long straight wire, infinite large plane, etc. In the part of steady magnetic field and electrostatic field, abstract concepts and models also have similarities, such as micro elementary charge is corresponding to the elementary current, infinite long straight charged conductor to infinite long straight current-carrying conductor, infinite charged plane to 
infinite current-carrying plane, etc. These are one-to-one correspondence, so we have to master the concepts in the electrostatic field when we understand the abstract concepts. Then the method of analogy can be used to deal with the corresponding concepts in the magnetic field to help students better understand and avoid the confusion with similar concepts.

3) It is conducive to improve students' learning enthusiasm

Due to the difficulty of electromagnetism course, if the teacher can not better process the knowledge points and choose appropriate teaching methods, it will lead to the result that teachers are performing singly on the platform and even part students cannot follow teachers. Others may do their own things for they aren't able to understand, which will badly affect the teaching quality. By using the idea of analogy method, teachers make an analogy between new content and involved similar content former. On the one hand, students may have the familiar feeling, which is helpful to reduce their fearing about the new knowledge; meanwhile, such method can improve their learning efficiency and help them quickly acquire knowledge to increase the achievement feeling and promote learning enthusiasm and initiative.

4) It is beneficial for students to apply what they have learned

Students should apply the knowledge they have learned to solve problems. The premise must be the full understanding and mastering of knowledge, and then they will be able to use it skillfully. Electromagnetism is a difficult course for students to learn. However, electromagnetism is a discipline closely related to our real life. For example, where there is electric current, there must be electromagnetic field. Our earth is a natural magnet, and life is full of electromagnetic waves. Meanwhile, we are also applying electromagnetic waves. Students can better understand the corresponding concepts and knowledge points and master the relationship among these knowledge points by using the idea of analogy method, so as to solve the corresponding problem and provide a basis for applying the theoretical knowledge they have learned to the real life.

\section{RESEARCH ON THE APPLICATION OF ANALOGY IN TEACHING}

\section{A. Analogy of conceptual understanding}

The analogy of conceptual understanding can help students remember, understand and master concepts accurately. The concepts in the electrostatic field and steady magnetic field mainly include the "ideal physical model", "basic characteristic physical quantity" and "imaginary curve".

\section{1) Analoqy of ideal physical model}

When introducing the electrostatic field and steady magnetic field, we should start with the basic concepts. In order to simplify the discussion, we introduce the ideal physical models of point charge and elementary current respectively. And the point charge only considers its charge, without considering its linearity and space volume. It is treated as a geometric point with no shape and size, only with electric quantity. For a general continuous charged body, the micro-charge is expressed in terms of $d q$. Elementary current, generally represented with $I d \vec{l}$, refers to a segment microelement by any electrically charged wire exciting the magnetic field in its surrounding space. And $d q$ is a scalar, Idl $\vec{l}$ is a vector.

2) Analogy of fundamental characteristics physical quantity

Electric and magnetic fields are special forms of matter existing in nature. They have strong effects on the charge put into them. In electric fields they are subjected to Coulombic force and Lorentz forces in magnetic fields. These two forces can be represented by the electric field intensity vector $\vec{E}$ and magnetic induction intensity vector $\vec{B}$ respectively. And these two physical quantities can also be used to describe the fundamental characteristics of electrostatic field and steady magnetic field. The field intensity excited by the microelement charge $d q$ in its surrounding space can be expressed as $d \vec{E}=\frac{d q}{4 \pi \varepsilon_{0} r^{2}} \vec{e}_{r}$, and the experiment shows that the field intensity is only related with the medium of field source $d q$, field point and surrounding space. Similarly, the magnetic induction intensity of the elementary current $I d \vec{l}$ can be expressed as $d \vec{B}=\frac{u_{0}}{4 \pi} \cdot \frac{I d \vec{l} \times \vec{e}_{r}}{r^{2}}$. We can find that the two expressions are similar in form, and the experiment shows that $\vec{B}$ is only related to the field source $I d \vec{l}$, field point and surrounding medium

\section{3) Analoqy of imaqinary curve}

Electric field and magnetic field are real materials in space, but they cannot be seen or touched. In order to give students a more intuitive description, we respectively introduced imaginary electric field lines and magnetic induction lines. By using such imaginary curves, we can also describe the space electric field and magnetic field. The density of the curve reflects the size of the field, and the arrow direction of the curve indicates the direction of the space field intensity. In specific, we can introduce the concept of electric flux, which means the line numbers of the unit area that are perpendicular to the field direction through some point, and we call it electric flux. It can be represented as the formula $\vec{E}=\frac{d \Phi_{e}}{d \vec{s}_{\perp}}$, and magnetic flux can be represented as the formula $\vec{B}=\frac{d \Phi_{B}}{d \vec{s}_{\perp}}$. The two formulas are very similar in form, and perfect effects can be achieved through the analogy method. They are also the important basis for obtaining Gaussian theorem in electric field and magnetic field respectively.

\section{B. Analogy of physical laws}

Electrostatic field and steady magnetic field are vector fields, and their main physical laws are on one law and two 
theorems. Therefore, in the process of learning, we find out a basic major experiment law and two theorems is the important premise of understanding the contents of this part. We still can use analogy thought to discuss one law and two theorems here.

1) The analogy of basic experimental laws

In electrostatic field, a fundamental experimental law refers to the Coulomb law, and the electric field strength formula of point charge can be derived: $d \vec{E}=\frac{d q}{4 \pi \varepsilon_{0} r^{2}} \vec{e}_{r}$, which is a differential type. If we want to discuss the electric field excited by any charged body in space, we still need to use field intensity superposition principle, and it can be obtained that $E=\int d \vec{E}=\int \frac{d q}{4 \pi \varepsilon_{0} r^{2}} \vec{e}_{r}$. In principle, we can find out the electric field of any charged body, but it is usually complicated due to the knowledge of vector and calculus. Similarly, the fundamental experimental law of steady magnetic field is Biot -- Savart law. As for the steady magnetic field excited by any elementary current in space, we can use the law to represent: $d \vec{B}=\frac{u_{0}}{4 \pi} \cdot \frac{I d \vec{l} \times \vec{e}_{r}}{r^{2}}$. It has the same status with Coulomb law. We still need to deal with differential expression, use the superposition principle and integral to get $\vec{B}=\int d \vec{B}=\int \frac{u_{0}}{4 \pi} \cdot \frac{I d \vec{l} \times \vec{e}_{r}}{r^{2}}$. It is usually difficult to calculate with this formula, because of the involved knowledge of vector and calculus.

\section{1) Analogy of Important theorems}

For the discussion of electrostatic field and vector property of steady magnetic field, we mainly start from two aspects: one is the flux of vector field through any closed surface, namely Gauss theorem; the other is the circulation of vector field along any closed path, namely Ampere circuital theorem. The two important theorems mentioned in electrostatic field and steady magnetic field are Gauss theorem and Ampere circuital theorem. In electrostatic field, Gauss theorem can be expressed as $\Phi_{e}=\iint_{s} \vec{E} \cdot d \vec{S}=\frac{\sum q_{i n}}{\varepsilon_{0}}$, and this equation reflects the nature of electrostatic field that is an active field, so its electric field line is not a closed curve. Ampere circuital theorem can be expressed as $\int_{l} \vec{E} \cdot d \vec{l}=0$, which reflects that the electric charge moves around any closed path, and the electric field force does no work, so the electric field force is conservative force. Electrostatic field is conservative field or non-rotating field. From Gauss theorem and Ampere circuital theorem, we can know that electrostatic field is active and non-rotating field. Similarly, we can use similar methods to analyze the steady magnetic field. Gauss theorem in the steady magnetic field can be expressed as $\Phi_{B}=\iint_{\mathrm{s}} \vec{B} \cdot d \vec{S}=0$. It reflects that the steady magnetic field is a passive field with no starting point and no ending point, that is, the magnetic induction line is a closed curve; Ampere circuital theorem can be expressed as $\int_{l} \vec{B} \cdot d \vec{l}=u_{0} \sum I_{i n}$, which reflects that the line integral of magnetic induction intensity around the closed path is equal to $u_{0}$ times of the algebraic sum of all current enclosed by the closed loop. Magnetic field force is non-conservative force, and the magnetic field is non-conservative field or has a spin field.

One law and two theorems of electrostatic field and steady magnetic field are respectively corresponding in the form, but in the specific application to solve the problem, Gauss theorem in the electrostatic field is corresponding with the circuital theorem in the magnetic field, and vice versa. In the later analogy of solution, we will make detailed introduction.

\section{2) Solution analogy}

Gauss's theorem and Ampere circuital theorem are two important theorems in electromagnetism, and we need to rely on them to solve problems. For some objects with symmetry, it will be very troublesome if we use the formula of field strength or Bio-Savart's law to solve the problem, but it is very simple to apply Gauss's theorem or the circuital theorem, and the solution and method are very similar. First, analyze the symmetry of the object to determine the symmetry of the field distribution, and then select the appropriate Gaussian surface or loop. Then the value of $\vec{E}$ at any point on the Gaussian surface is same, or its direction is parallel or perpendicular to the Gaussian surface; in the selected loop in the steady magnetic field, it is required that the value of $\vec{B}$ is same everywhere, or its direction is parallel or perpendicular to the loop. At this point, Gauss's theorem in the electrostatic field can be simplified into $E S=\frac{\sum q_{i n}}{\varepsilon_{0}}$, and the circuital theorem can be simplified to $B l=u_{0} \sum I_{\text {in }}$ in the steady magnetic field. We can find out the value of $\vec{E}$ and $\vec{B}$ as long as we know the area of Gaussian surface $S$ and the length of the loop $l$ as well as the algebraic sum of surrounding charge and electric current. While the surface and loop are made by us, and they are regular, so they are very easy to get. Now we use Gauss's theorem and circuital theorem to illustrate.

Example 1: Suppose there is a straight wire with infinite length in the vacuum, and the charge is uniformly distributed, and the linear density is $\lambda$, then calculate the electric field intensity at the distance of $r$ from the direct wire.

Solution: Since the object of study is axisymmetric, the electric field is also axisymmetric. We can choose a concentric cylinder surface with any point of the straight wire as the center of the circle, $r$ as the radius, and $\pi r^{2}$ as the base area as well as $h$ as the height. According to the Gauss's theorem of electrostatic field $\mathscr{S}_{s} \vec{E} \cdot d \vec{S}=\frac{\sum q_{i n}}{\varepsilon_{0}}$, it can be obtained that: 


$$
\begin{aligned}
& \oiiint_{s} \vec{E} \cdot d \vec{S}=E \cdot S=\frac{\sum q_{i n}}{\varepsilon_{0}} \\
& \Rightarrow E \cdot 2 \pi r h=\frac{\lambda h}{\varepsilon_{0}} \\
& \Rightarrow E=\frac{\lambda}{2 \pi \varepsilon_{0} r}
\end{aligned}
$$

Example 2: Assuming that there is a uniformed long current conductor with infinite length, the intensity of electric current is $I$, then calculate the magnetic induction intensity at the distance of $r$ from the current-carrying straight conductor.

Solution: Since the object of study is axisymmetric, the magnetic field is also axisymmetric. We can select any point on the straight wire as the center of the circle and make $r$ as the radius to make a circle as the ampere loop. According to the circuital theorem of steady magnetic field $\oint_{l} \vec{B} \cdot d \vec{l}=u_{0} \sum I_{\text {in }}$, it can be obtained that

$$
\begin{aligned}
& \oint_{l} \vec{B} \cdot d \vec{l}=B \cdot l=u_{0} \sum I_{\text {in }} \\
& \Rightarrow B \cdot 2 \pi r=u_{0} I \\
& \Rightarrow B=\frac{u_{0} I}{2 \pi r}
\end{aligned}
$$

From the discussion of the two typical examples above, we can find out the correspondence and similarity in solution thinking and method steps between Gauss's theorem and circuital theorem in electrostatic field and steady magnetic field. By grasping the above characteristics and using analogy thought, we can help students better understand and apply the two theorems to solve the problem.

\section{CONCLUSION}

In conclusion, electrostatic field and steady magnetic field are closely related, both the arrangement of the content sequence and the strong symmetry in knowledge points and mathematical treatment methods. Therefore, when teaching this part of content, we should timely apply the idea of analogy, which can accelerate students' understanding and connection with the new concepts and rules, master new knowledge and apply new knowledge to solve problems, so as to stimulate their enthusiasm and initiative in learning. At the same time, the introduction of analogy idea can help students establish a clear knowledge frame to integrate the knowledge thoroughly and make it easy to remember. And it is helpful to cultivate students' scientific thinking abilities and innovation abilities.

\section{REFERENCES}

[1] Liang Canbin. Electromagnetism[M]. Beijing: Advanced education press,2012.1-213.

[2] Huang Wuying. Exploration on the teaching reform of electromagnetism of physics major in normal universities[J]. College Physics, 2017.(5):27-28.

[3] Yin Cailiu. The application of analogy method in the electromagnetism teaching of College Physics[J]. Journal of Guangxi University,2011(17):98-100.

[4] Tang Weiya. Research on the application of analogy method in electromagnetism teaching [J]. Journal of higher education, 017(2): 6-97.

[5] Zhang Xiangui. Research on the application of analogy method in electromagnetism teaching[J]. Journal of Agricultural University of Hebei,2015(6):109-111.

[6] Wen Nai. The application of analogy method in electromagnetism teaching[J]. Physics Bulletin,2013(6):11-12. 